\title{
Building a biopsychosocial model of cancer-related fatigue: the BIOCARE FActory cohort study protocol
}

\author{
M. Chartogne ${ }^{1 *}$ (D) A. Leclercq ${ }^{1}$, B. Beaune ${ }^{1}$, S. Boyas ${ }^{1}$, C. Forestier ${ }^{1}$, T. Martin' ${ }^{1}$, V. Thomas-Ollivier², S. Landry ${ }^{3}$, \\ H. Bourgeois ${ }^{3}$, O. Cojocarasu', V. Pialoux ${ }^{5}$, O. Zanna ${ }^{6}$, L. A. Messonnier ${ }^{7}$, A. Rahmani ${ }^{1 \dagger}$ and B. Morel ${ }^{1,7 \dagger}$
}

\begin{abstract}
Background: Cancer-related fatigue (CRF) is the most common side effect of cancer and cancer treatment. CRF prevalence is up to 50\% in breast cancer patients and can continue several years after cancer remission. This persistent subjective sense of exhaustion is multifactorial. Numerous parameters have been evidenced to be related to CRF across biological, physical, psychological, social and/or behavioral dimensions. Although CRF has been studied for many years, the majority of previous studies focused on only one dimension, i.e., physical function. Moreover, few studies investigated CRF longitudinally with repeated measures. These are the two main obstacles that limit the understanding of CRF mechanisms. The purpose of this study is to create a biopsychosocial model of CRF with simultaneous and longitudinal anthropometric, clinical, biological, physical, psychological and sociological parameters.
\end{abstract}

Methods: BIOCARE FActory is a multicentric prospective study that will consist of an 18-month follow-up of 200 women diagnosed with breast cancer. Four visits will be scheduled at diagnosis, after treatments, and 12 and 18 months after diagnosis. The same procedure will be followed for each visit. Each session will be composed of anthropometric data collection, a semi-structured interview, cognitive tests, postural control tests, neuromuscular fatigability tests and a cardiorespiratory fitness test. Clinical and biological data will be collected during medical follow-ups. Participants will also complete questionnaires to assess psychological aspects and quality of life and wear an actigraphy device. Using a structural equation modeling analysis (SEM), collected data will build a biopsychosocial model of CRF, including the physiological, biological, psychological, behavioral and social dimensions of CRF.

Discussion: This study aims to highlight the dynamics of CRF and its correlates from diagnosis to post treatment. SEM analysis could examine some relations between potential mechanisms and CRF. Thus, the biopsychosocial model will contribute to a better understanding of CRF and its underlying mechanisms from diagnosis to the aftermaths of cancer and its treatments.

Trial registration: This study is registered at ClinicalTrials.gov (NCT04391543), May 2020.

\footnotetext{
* Correspondence: martin.chartogne@univ-lemans.fr

${ }^{\dagger}$ A. Rahmani and B. Morel contributed equally to this work.

'Le Mans Université, Movement - Interactions - Performance, MIP, 4334,

F-72000 Le Mans, EA, France

Full list of author information is available at the end of the article
}

(c) The Author(s). 2021 Open Access This article is licensed under a Creative Commons Attribution 4.0 International License, which permits use, sharing, adaptation, distribution and reproduction in any medium or format, as long as you give appropriate credit to the original author(s) and the source, provide a link to the Creative Commons licence, and indicate if changes were made. The images or other third party material in this article are included in the article's Creative Commons licence, unless indicated otherwise in a credit line to the material. If material is not included in the article's Creative Commons licence and your intended use is not permitted by statutory regulation or exceeds the permitted use, you will need to obtain permission directly from the copyright holder. To view a copy of this licence, visit http://creativecommons.org/licenses/by/4.0/. The Creative Commons Public Domain Dedication waiver (http://creativecommons.org/publicdomain/zero/1.0/) applies to the data made available in this article, unless otherwise stated in a credit line to the data. 
Keywords: Cancer-related fatigue, Breast cancer, Fatigability, Correlates of fatigue, Structural equation modeling, Follow-up

\section{Background}

Cancer and cancer treatments induce various sideeffects, the most reported being cancer-related fatigue (CRF). CRF is defined as 'a distressing persistent subjective sense of physical, emotional, and/or cognitive tiredness or exhaustion related to cancer or cancer treatment that is not proportional to recent activity that interferes with usual functioning' [1]. In breast cancer patients, CRF prevalence is up to $50 \%$ and can persist two years after cancer remission for $30 \%$ of patients and 5 years after for $20 \%$ [2]. CRF is extremely disturbing to quality of life and can decrease survival (overall and recurrencefree survival) [3]. Numerous parameters have been evidenced to be related to CRF among biological, physical, behavioral, psychological and/or social dimensions [4] (Table 1). Below is a summary of the main correlates of CRF (for extensive reviews see 5,6,7).

Anthropometric data such as BMI or age have been, respectively, positively and negatively correlated to CRF severity [5-8]. Clinical data such as treatment type and disease stage have been associated with higher severity of CRF [9-11]. This relationship, however, remains unclear, and Bower et al., [12] concludes that treatment-related factors accounted for only a small portion of CRF.

Biological parameters extracted from blood samples, such as inflammation and anemia, have been related to CRF in breast cancer survivors through elevated levels of pro-inflammatory cytokines (e.g., TNF- $\alpha$, IL6) and reduced hemoglobin levels, respectively [13, 14].

Regarding physical parameters, CRF patients are more susceptible to sarcopenia, a substantial loss of skeletal muscle, thereby altering muscle strength and endurance [15]. This decrease in the force-generating capacity of the neuromuscular system during exercise, or neuromuscular fatigability, is higher in cancer patients suffering from CRF and is probably controlled by a specific central etiology [16-20]. Cardiorespiratory deconditioning is also linked to CRF; $\mathrm{VO}_{2 \text { peak }}$ and power output at the lactate threshold have been correlated to CRF's severity in cancer survivors [21], possibly due to the cardiac toxicity of some chemotherapies [22].
Behavioral parameters such as sleep disturbances are highly prevalent (up to 70\%) in patients experiencing CRF [23]. Recent objective measures of sleep showed that onset latency, wake time at night, and sleep efficiency were correlated with CRF severity [24]. Lastly, duration and intensity of spontaneous daily physical activities are also related to CRF, as patients show an increase in leisure time and low intensity activities $(<2.5$ metabolic equivalent task, METs) compared to those without CRF [25].

The relationship to psychological dimensions is also well established. Using specific questionnaires, anxiety and depression were investigated in breast cancer patients across many years and were strongly correlated with CRF severity [26]. Coping strategies, particularly catastrophizing (a lack of confidence and an expectation of negative outcomes), may be associated with CRF [27]. Furthermore, women with breast cancer may undergo surgical mastectomy in addition to chemotherapy, which impacts body image, self-esteem and may lead to depressive symptoms $[28,29]$. Cognitive processes, such as attention, concentration and memory, are affected before and during treatments and related to CRF [30]. These cancer-related cognitive impairments have been frequently attributed to chemotherapy neurotoxicity and dubbed "chemo fog" or "chemobrain" [31]. As part of the social dimension of CRF, social networks and support were studied using questionnaires and interviews. Generally, the lack of social support was identified as a factor of fatigue in chronic fatigue syndrome [32]. Among breast cancer patients, those who reported lower levels of social support had elevated CRF [33].

Despite the numerous studies investigating CRF correlates, including their potential mechanisms and the well-known multidimensional nature of CRF, the majority of previous studies have remained mainly focused on a specific dimension (e.g., biological or psychological). This is a significant limitation to understanding the relationships between all of the known correlates of CRF. There are, however, a few studies that have combined some of the above-mentioned dimensions in order to investigate CRF mechanisms. For example, Stone et al., [34] designed a model using a multiple

Table 1 Hypothesized mechanisms of CRF and corresponding dimensions (adapted from McNeely and Courneya 2010)

\begin{tabular}{llll}
\hline Physiological & Biological & Psychological & Behavioral \\
\hline - Muscular strength & - Inflammatory response & - Anxiety & . Sleep quality and quantity \\
- Muscular endurance & - Metabolic function & • Depression & • Appetite \\
- Cardiopulmonary fitness & - Endocrine function & - Distress & \\
- Body composition & $\cdot$ Immune function & - Cognition & \\
\hline
\end{tabular}


linear regression, explaining $56 \%$ of CRF variance including anxiety-depression, dyspnea and pain, and a disease burden score. More recently, Lockefeer et al., [26] used depressive symptoms, sleep quality and CRF before diagnosis (or primary surgical treatment) in breast cancer patients to predict CRF at 24 months. Only CRF before diagnosis was a significant predictor of CRF two years later and explained 33\% of CRF variance. Humpel et al., [35] also investigated the relationship between sleep disturbances, CRF and physical activity in patients with breast cancer. Their CRF prediction model included sleep quality and total physical activity and resulted in a $46 \%$ CRF variance prediction. Nevertheless, they focused exclusively on the behavioral dimensions of CRF. Recently, CRF prediction has been studied by combining neuromuscular, emotional and behavioral dimensions [20]. The results evidenced that a model including anxietydepression, sleep disturbances and neuromuscular fatigability explained $56 \%$ of CRF variance. CRF variances described by the above-mentioned studies ranged from 33 to $56 \%$, leaving unexplained variations which could be related to dimensions not yet considered.

Most studies investigating CRF were cross-sectional and thus cannot account for the dynamics of these mechanisms, and few studies implemented longitudinal follow-ups. Some longitudinal studies have focused on the psychological dimension and their results are unanimous in asserting that anxiety before treatment was a strong predictor of subsequent CRF in breast cancer patients [26, 36, 37]. At best, these various studies combine only two different dimensions (behavioral and psychological, biological and psychological, clinical and psychological, respectively). Only Bower et al., [5] have led a longitudinal study in breast cancer patients that combines more than two dimensions of CRF, from diagnosis to 18 months post treatment. They have investigated biological, demographic, social, clinical and psychological dimensions. Unfortunately, only baseline results are currently available. They reveal that younger age, lower educational level, lower disease stage and history of childhood maltreatment were found to be significant predictors of CRF.

Recent studies have highlighted the necessity to develop longitudinal and multidimensional researches in order to identify potential mechanisms explaining CRF $[8,38]$. Therefore, the purpose of this longitudinal study is to build a biopsychosocial model of CRF by simultaneously investigating anthropometric, clinical, biological, physical, psychological and sociological parameters using structural equation modeling (SEM).

\section{Methods/design \\ Study population}

Two hundred women newly diagnosed with breast cancer by an oncologist and satisfying inclusion and exclusion criteria (Table 2) will be informed of the study protocol. If interested, patients will receive an information letter from their oncologist and will be given the opportunity to ask any questions pertaining to it. After a $48 \mathrm{~h}$ period of reflection, study coordinators will phone the patients to confirm their participation, obtain a written consent form and schedule their first visit.

\section{Study design}

This prospective multicentric (Clinique Victor HugoCentre Jean Bernard, Le Mans, France and Centre Hospitalier du Mans, Le Mans, France) study will consist of an 18 month follow-up of a women cohort with breast cancer. Four visits will be scheduled (the study timeline is presented in Fig. 1). Detailed patient assessments will be performed before treatment begins (visit 1: diagnosis);

Table 2 Inclusion and exclusion criteria

\begin{tabular}{|c|c|}
\hline \multirow[t]{7}{*}{$\begin{array}{l}\text { Inclusion } \\
\text { criteria }\end{array}$} & $\begin{array}{l}\text { - Patients care by chemotherapy or radiotherapy in Clinique Victor Hugo-Centre Jean Bernard, Le Mans, France or in } \\
\text { Centre Hospitalier du Mans, Le Mans, France }\end{array}$ \\
\hline & - Patients with breast cancer diagnosis (Stage I to IIIc) \\
\hline & - Aged $\geq 18$ and $\leq 80$ years \\
\hline & - Approval received from oncologist \\
\hline & - ECOG Performance Status $\leq 2$ \\
\hline & - French speaking (able to understand questionnaires and instructions related to study procedures) \\
\hline & - Written informed consent obtained \\
\hline \multirow{7}{*}{$\begin{array}{l}\text { Exclusion } \\
\text { criteria }\end{array}$} & - Comorbidities related to fatigue symptoms \\
\hline & - Polyneuropathy, amyotrophy or myasthenic syndrome diagnosis \\
\hline & - Contraindications to physical activity or to experimental procedures \\
\hline & - Antidepressants, psychostimulants, psychotropics, antiépileptics or benzodiazepine based treatment \\
\hline & - Previous or current psychosis, bipolarity or severe depression symptoms \\
\hline & - History of chronic fatigue, stroke or musculoskeletal disorders \\
\hline & - Participant is pregnant \\
\hline
\end{tabular}




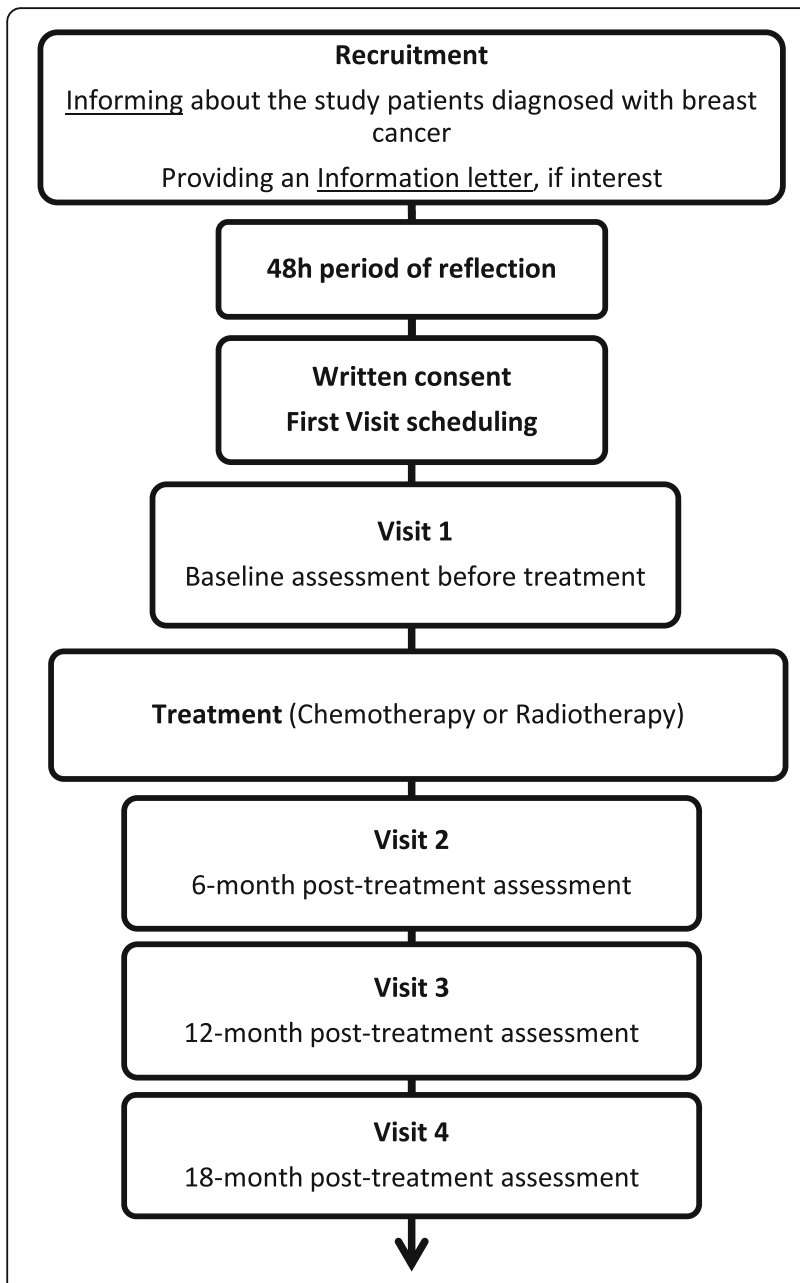

Fig. 1 Flowchart of the study design

during the week after completion of the first-line treatment, or 6 months after diagnosis, whichever comes first (visit 2); and then 12 and 18 months after diagnosis (visit 3 and 4, respectively). This study has been approved by the French ethics committee of human research CPP SUD EST VI (IDRCB: 2019-A02525-52) and will be performed according to the Declaration of Helsinki. Furthermore, this study protocol has been written in accordance with the SPIRIT guidelines (SPIRIT Checklist provided in Additional file 1) and is registered in a database (ClinicalTrials.gov, NCT04391543, May 2020). In order to longitudinally assess the evolution of biopsychosocial dimensions of CRF, the same procedure will be followed for each visit. Each session will last $1.5 \mathrm{~h}$ and will be composed of anthropometric data collection, a semi-structured interview, cognitive tests, postural control tests, neuromuscular fatigability tests and a cardiorespiratory fitness test. Clinical and biological data will be collected during medical follow-ups. In addition, participants will be asked to wear an actigraphy device (received by mail one week prior to each experimental session) and to complete questionnaires to assess psychological aspects and quality of life.

\section{Anthropometric data}

Body height, body mass, lower limb fat mass (using impedancemetry) and calf circumference (CC) will be measured. $\mathrm{CC}$ will be recorded to assess the dominant lower limb volume using the truncated cones technique [39] by dividing the lower limb volume into a series of segments. Then, the lower limb volume will be used to normalize maximal strength of participants. CC under $31 \mathrm{~cm}$ will serve as a clinical indicator of sarcopenia [40].

\section{Semi-structured interview}

To investigate the social dimension of CRF, a semistructured interview will be conducted (duration $\sim 30$ min). Demographic (e.g., age, gender, socio-professional category, residence area, income) and sociability (e.g., family, friendly, professional) will be addressed. The interview will be introduced by an open question on a typical week to then develop the sociability. The entire interview will be audio recorded, fully transcribed and then will be the subject of a lexicographical treatment for rating quantity and intensity of sociability (on a scale from -5 to 5). In order to build a typology of patients' sociability, researchers will also assess the level of social ties' perception. Lastly, family, friendships, professional sociabilities and income will be considered in the SEM analysis.

\section{Cognitive tests}

The Montreal Cognitive Assessment (MoCA), the Trail Making Test (TMT) and the Stroop test will be completed by participants to assess cognitive functions. The MoCA is separated into several tasks including visuospatial/executive functioning, naming, memory, attention, language, abstraction, delayed recall and orientation. For example, the participants will be asked to connect numbers and letters in a defined sequence, count backwards from 100 by increments of 7, draw different figures and perform word associations. The MoCA total score (/30) is obtained by summing the scores from each item; a cut-off score below 26 is an indicator of mild cognitive impairment [41]. The TMT, evaluating processing speed and cognitive flexibility, consists of connecting numbers in ascending order (Part 1 ) and then in connecting numbers and letters in both ascending and alphabetical order (Part 2), as fast as possible [42]. Time needed to complete each part and number of mistakes will be recorded to assess performance. The Stroop test is based on color recognition (i.e., red, green, yellow and blue) with interference in three parts, each composed of 6 lines with 4 items per line (colored sticker or word). The first part is composed of colored stickers (control), the second has colored words (low interference) 
and the last part has color-words printed in a color not denoted by the name (high interference). For each part, participants will be advised to name the ink color for each word, regardless of the semantic content, as quickly and accurately as possible. The time to complete each part is measured to attribute an interference score used to assess response inhibition, which has been related to cognitive functioning in daily life [43]. The TMT and the Stroop test will be implemented on Inquisit software (Millisecond Software, LCC, Seattle, USA). For SEM analysis, MoCA total scores (/30), TMT time (s) and Stroop interference scores (\%) will be considered.

\section{Postural tests}

Participants will be asked to stand up barefoot, as still as possible, during $60 \mathrm{~s}$ on a pressure distribution measurement platform (FDM-S, zebris Medical GmbH, Isny, Germany). Feet position will be standardized using markings to keep both heels spaced apart by $10 \mathrm{~cm}$ and an angle of $15^{\circ}$ between both feet. Participants will be advised to keep arms alongside the body during recording. Four conditions composed of two trials of a simple task and two trials of a dual task (counting backwards by 2 from a number close to 100) with eyes open or closed will be counterbalanced and interspersed with a $2 \mathrm{~min}$ rest period. In eyes open conditions, participants will be asked to stare at a visual marker placed $2 \mathrm{~m}$ in front at eye level. For the dual task, participants will be asked to count as fast as possible whilst remaining as immobile as possible. The numbers of answers and errors will be recorded. Temporal analysis will be performed in order to investigate anterior-posterior and medial-lateral center of pressure (COP) distance, mean and maximal COP velocities and 95\% confidence ellipse area. In addition, the frequency domain measures (relative power in $<0.5$ $\mathrm{Hz} ; 0.5-1.5 \mathrm{~Hz} ;>1.5 \mathrm{~Hz}$ frequency bands) will be computed. $95 \%$ confidence ellipse area $\left(\mathrm{mm}^{2}\right)$ will be considered in the SEM to represent the physical function latent variable, while maximal COP velocities $(\mathrm{mm} / \mathrm{s})$ will be considered representative of the emotional function latent variable, as it is a relevant hallmark of depression-related psychomotor retardation [44].

\section{Neuromuscular fatigability test}

Throughout this test, participants will remain in prone position on a patient table, with a fullyextended knee and an ankle angle of $90^{\circ}$, their foot securely blocked at the metatarsal level in a custommade device enabling isometric strength measurement with a load cell (LSB350, Futek, Irvine, USA). Firstly, optimal electrical stimulation intensity will be determined to set the supramaximal intensity used during the subsequent neuromuscular assessments by progressively increasing the current (from 20-mA to 200-
$\mathrm{mA}$, with a 20-mA increment) until there is no further increase in the evoked isometric twitch response. The last intensity obtained will be further increased by $20 \%$ to ensure stimulus supramaximality. All electrical stimulations will be delivered with a constant current (Digitimer DS7A-H, Hertfordshire, UK) using square-wave stimuli of $200 \mu$ s duration with a maximal voltage of $400-\mathrm{V}$ and via rectangular selfadhesive electrodes $(5 \times 10 \mathrm{~cm}$, Compex). The cathode will be placed over the gastrocnemii $(\sim 5 \mathrm{~cm}$ distal to the popliteal fossa) and the anode over the soleus ( $10 \mathrm{~cm}$ proximal to the calcaneus). Secondly, participants will accomplish a standardized warm-up (10 isometric contractions of $4 \mathrm{~s}$ at $50 \%$ of their maximal strength with $4 \mathrm{~s}$ of recovery in between), followed by 2-min of rest and then by the maximal voluntary contraction (MVC) measurement (two MVC of $4 \mathrm{~s}$ separated by $2 \mathrm{~min}$; if the difference between these MVCs is superior to $5 \%$, a third one will be performed). The higher peak force produced will be considered as the MVC in non-fatiguing conditions. Next, pre-fatigue neuromuscular functions will be assessed on a third MVC, using a $100-\mathrm{Hz}$ doublet during the force plateau and a stimulation sequence on the relaxed muscles beginning $2 \mathrm{~s}$ after the end of contraction: a 100$\mathrm{Hz}$ doublet, a $10-\mathrm{Hz}$ doublet and a simple stimulation, interspersed by $3 \mathrm{~s}$. The fatiguing exercise will be composed of 62 isometric MVC in ankle plantar flexors with the dominant leg (Fig. 2). Each MVC will last $4 \mathrm{~s}$ with $1 \mathrm{~s}$ rest; duty cycle will be ensured using a metronome with visual and sound signals. To avoid pacing strategies, participants will not be informed of the time remaining or the number of MVC performed [45]. Investigators will use verbal encouragements for participants to contract as strong as possible during MVC. The post-fatigue neuromuscular functions will be assessed on the last MVC of the fatiguing exercise (62nd) with the same stimulation procedure as in pre-fatigue condition. On the 60th $\mathrm{MVC}$, neuromuscular functions will also be tested but only with two $100-\mathrm{Hz}$ doublets (during the force plateau and on the relaxed muscles $2 \mathrm{~s}$ after the MVC end).

Peak force occurring during each 4-s MVC set will be recorded and the force-time relationship asymptote $\left(\mathrm{F}_{\mathrm{A}}\right.$; expressed in percentage of the MVC in nonfatiguing conditions) will be used to represent a neuromuscular fatigability threshold, above which fatigability drastically sets in $[20,46,47]$. Then, voluntary activation (VA) and evoked forces by a $100-\mathrm{Hz}$ doublet (Db100) will be determined using the interpolated twitch technique [48] and expressed in pre-post fatiguing test differences, normalized to pre-fatiguing test values. $\mathrm{F}_{\mathrm{A}}$ (\% MVC), VA (\%) and Db100 (\%) will be considered in the SEM. 


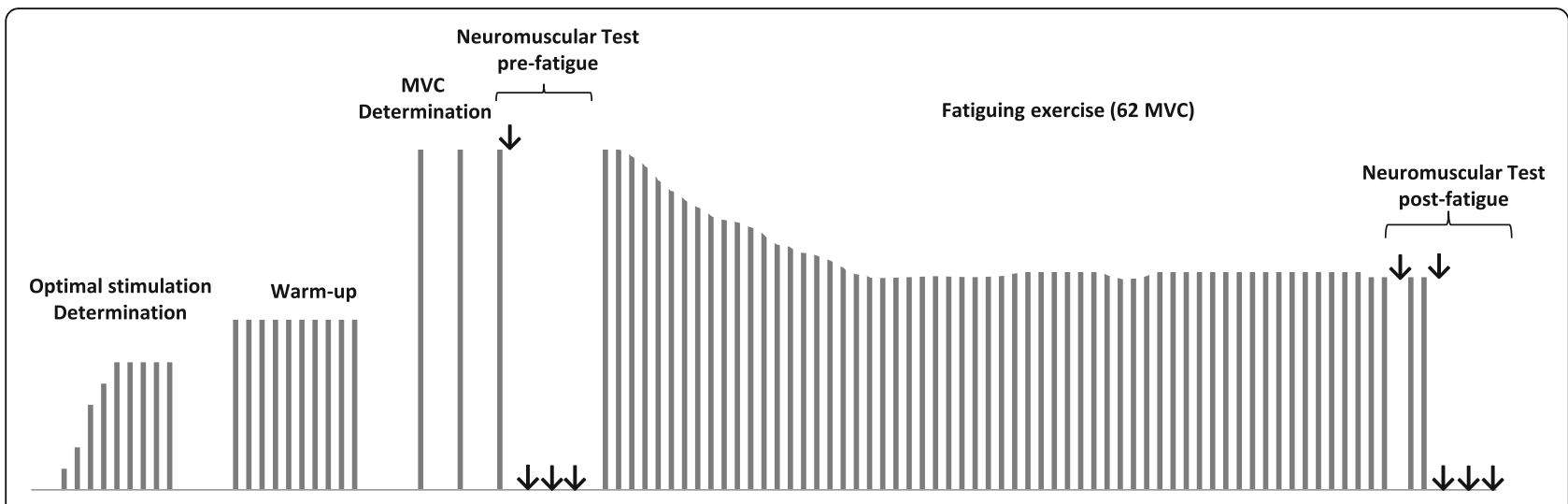

Fig. 2 Schematic illustration of the fatiguing exercise and neuromuscular assessments. $\downarrow$ represents electrical stimulation

\section{Cardiorespiratory fitness test}

After a five-minute recovery period, a blood drop $(10 \mu \mathrm{L})$ will be taken from the earlobe and analyzed extemporaneously (within $15 \mathrm{~s}$ ) to obtain a resting blood lactate concentration $\left([\text { Lactate }]_{\mathrm{b}}\right.$ ) (Lactate Scout 4, EKF diagnostics, Cardiff, UK). Then, participants will perform a submaximal incremental exercise on a cycle ergometer (model 928E, Monark, Varberg, Sweden), with saddle and handlebar heights adjusted for each patient. Throughout the test, participants will be encouraged to maintain a constant cadence (approximately $60 \mathrm{rpm}$ ) and heart rate (HR) will be recorded with a heart rate monitor (HRM-Dual, Garmin, Olathe, USA). After a 3min standardized warm-up at a rate of perceived exertion (RPE) of 8-9 (Borg 6-20 scale), the test will start at $20 \mathrm{~W}$. Every 2 min [Lactate $]_{\mathrm{b}}$ will be measured as previously described and then intensity will be increased by $10 \mathrm{~W}$ steps. Just before the end of the step, participants will indicate RPE (Borg 6-20 scale). Exercise will be stopped as soon as i) [Lactate] $]_{\mathrm{b}} \geq 4 \mathrm{mmol}$. $\mathrm{L}^{-1}$; ii) participant RPE $>15$; or iii) participant will no longer be able to maintain the $60 \mathrm{rpm}$ cadence. Participants will be able to interrupt the exercise when they wish to do so, particularly in the case of nausea, chest pain or dyspnea. This test will be used to determine the physiological and biomechanical parameters (i.e., HR, power output associated with the first lactate threshold (LT1; defined as the first inflection point in the lactate concentration ([Lactate $]_{\mathrm{b}}$ ) curve). Power output at LT1 (W) will be considered in the SEM.

\section{Clinical and biological data}

As experimental sessions will accompany medical follow-ups, clinical and biological data will be also recorded. Cancer stage and details related to treatments (e.g., type, duration and dose) will be recorded in a medical file by their oncologist. Venous blood samples will be collected by medical staff. Plasma will be obtained after a $10 \mathrm{~min}$ centrifugation at $4{ }^{\circ} \mathrm{C}$, then divided into aliquots and stored at $-80{ }^{\circ} \mathrm{C}$ until analysis. Following assays will be performed on plasma for the assessment of inflammation (IL-6, TNFa, IL-8, IL-1 $\beta$ ). Abdominal computed tomography $(\mathrm{CT})$ images at the L3 level will be taken by medical staff on two occasions (Visit 1 and Visit 2). The surface of the muscular tissues will be selected according to the CT Hounsfield unit and normalized to stature in order to calculate the lumbar skeletal muscle index (LSMI). A cut-off value (LSMI $<38.5 \mathrm{~cm}^{2} /$ $\mathrm{m}^{2}$ ) will be used to characterize sarcopenia [49]. Cancer stage and treatment; sarcopenia; and IL-6, IL-1 $\beta$, TNFa $(\mathrm{pg} / \mathrm{mL})$ concentrations will be considered in the SEM.

\section{Participant-reported outcomes}

One week prior to each experimental session, participants will receive by post self-assessment questionnaires about quality of life (EORTC QLQ-C30), CRF (EORTC QLQ-FA12), coping strategies (Brief Cope) and anxietydepression symptoms (The Hospital Anxiety and Depression Scale - HADS). Participants will be asked to complete questionnaires, alone in quiet conditions, and bring it back the day of the experimental session. The subsequent instructions will be provided: "Please answer all questions yourself by circling the number that best applies to you. The information provided will remain strictly confidential. Take as much time as necessary. There are no "right" or "wrong" answers."

The EORTC QLQ-C30 Fatigue scale score (FA item) will be used to assess the general degree of CRF (ranging from 0 to 100; with higher levels indicating a greater degree of CRF), with a threshold for clinical importance of 39 [50]. EORTC QLQ-FA12 subscales will be used to assess physical, cognitive and emotional dimensions of CRF [51]. The HADS subscale scores (/21) will be used to assess anxiety and depression, respectively [52]. In the SEM, general degree of CRF (\%); dimensions of CRF (\%) (physical, cognitive and emotional); coping scores (/8); and anxiety and depression scales (/21) will be considered. 


\section{Actigraphy}

One week prior to each experimental session, participants will be asked to wear a portable device (eTact ${ }^{\circ}$, BodyCap, Caen, France) containing a tri-axial accelerometers enabling to record every acceleration from the body (sampling frequency: $25 \mathrm{~Hz}$, measurement range: $0.1-2 \mathrm{G}$, sample measurement: $1 \mathrm{~min}$ ). They will be advised to wear the device continuously for 7 days (days and nights) regardless of daily activities. Sleep characteristics will be obtained using the eTact ${ }^{\bullet}$ Analysis software (BodyCap, Caen, France), which assess the individual sleep quality based on the actigraphy data collected over selected periods. Considered sleep parameter will be sleep time (i.e, duration of effective sleep period) and sleep efficiency (i.e., percentage of time spent sleeping during the rest period). From the software, total activity duration during wake period will be calculated as the accumulated time in each activity intensity band (i.e., low, moderate and intense). In addition, participants will be asked to complete a sleep diary (including estimated sleep onset and offset hour) during the actigraphy measurements. Total activity duration (min) and sleep efficiency (\%) will be considered in the SEM.

\section{Sample size}

A sample size of $n=200$ patients has been chosen on the basis of resource constraints [53]. 200 correspond to a $15 \%$ inclusion rate of the patients meeting the inclusion criterion during a 24 months inclusion period and including a $20 \%$ potential drop-out rate. Considering this sample size, a sensitivity power analysis performed with G-power ( $F$ tests family, linear multiple regression, $R^{2}$ increase) to estimate the smallest effect size we could detect with $90 \%$ power regarding our main independent variable (i.e., CRF) at the level of analysis with the lowest power (i.e., level 2, between-person level). This analysis, with a power of 0.90 ,

Table 3 Latent and manifest variables included in the model built by SEM

\begin{tabular}{|c|c|c|}
\hline Latent variable & Manifest variable & Test \\
\hline \multirow[t]{4}{*}{ Social function } & Family sociability & Semi-structured interview \\
\hline & Friendly sociability & Semi-structured interview \\
\hline & Professional sociability & Semi-structured interview \\
\hline & Income & Semi-structured interview \\
\hline \multirow[t]{3}{*}{ Cognitive function } & MoCA total score & MoCA \\
\hline & TMT time & TMT \\
\hline & Interference score & Stroop \\
\hline \multirow[t]{8}{*}{ Physical function } & 95\% confidence ellipse area & Postural test \\
\hline & $\mathrm{F}_{\mathrm{A}}$ & Neuromuscular fatigability test \\
\hline & VA & Neuromuscular fatigability test \\
\hline & Db100 & Neuromuscular fatigability test \\
\hline & Power output at LT1 & Cardiorespiratory fitness test \\
\hline & Sleep efficiency & Actigraphy \\
\hline & Total activity duration & Actigraphy \\
\hline & Sarcopenia & Abdominal $\mathrm{CT}$ at the $\mathrm{L} 3$ level \\
\hline \multirow[t]{5}{*}{ Biological function } & Cancer stage & Medical file \\
\hline & Cancer treatment & Medical file \\
\hline & IL-6 concentration & Blood sample \\
\hline & IL-1 $\beta$ concentration & Blood sample \\
\hline & TNFa concentration & Blood sample \\
\hline \multirow[t]{4}{*}{ CRF } & FA item score & EORTC QLQ-C30 \\
\hline & Physical subscale & EORTC QLQ-FA12 \\
\hline & Cognitive subscale & EORTC QLQ-FA12 \\
\hline & Emotional subscale & EORTC QLQ-FA12 \\
\hline \multirow[t]{4}{*}{ Emotional function } & Coping score & Brief Cope \\
\hline & Anxiety scale & HADS \\
\hline & Depression scale & HADS \\
\hline & Maximal COP velocity & Postural test \\
\hline
\end{tabular}


an $\alpha$ level of 0.05 and 6 predictors (i.e., 6 latent variables), revealed that the smallest effect size we could detect with $90 \%$ power, at this level of analysis, is $f^{2}=0.09$, which correspond to a small to medium effect.

\section{Statistical analysis}

Hypotheses will be addressed by constructing a biopsychosocial model of the relationships among biological, physical, emotional, cognitive and social dimensions of CRF using multilevel SEM. SEM is particularly wellsuited to this type of analysis because it simultaneously accounts for multiple interactive relationships among variables, easily handles multiple sources of variance, and permits testing of hypothesized directional relationships $[54,55]$. Consequently, the results of SEM analyses facilitate inferences regarding the relationships among variables [56]. Relations between the manifest variables and their underlying latent constructs in a hypothesized biopsychosocial model predicting CRF are presented in Table 3 . The model will be tested with the maximum likelihood method using Lavaan $R$ package for SEM [57]. A primary cross-sectional analysis will be completed using data collected at each visit, and then subsequently by using the longitudinal data set. The models' fit was assessed by examining the minimum discrepancy (CMIN/DF), the probability level ( $p$-value), the BentlerBonett normed fit index (NFI), the comparative fit index (CFI), the Tucker-Lewis-Index (TLI), and the rootmean-square error of approx- imation (RMSEA). A satisfactory model fit is indicated by a CMIN/DF ratio below 2.00 [58], a $p$-value over 0.05 [59], a NFI over 0.95 [60], a TLI over 0.90 [61], a CFI over 0.93 [62], and a RMSEA below 0.05 [63]. All non-significant paths were deleted according to the methods described by MacCallum [64].

\section{Discussion}

American and European guidelines [65-67] recommend screening for CRF using self-reported questionnaires [68]. Even though this method remains simple and easily feasible for both clinician and patients, this assessment method does not enable the understanding and management of underlying mechanisms. Despite that CRF has been studied for decades, and recent studies have recognized that CRF is multifactorial and may be influenced by a variety of mechanisms $[8,38,69]$, few longitudinal and multidimensional studies have been implemented. This is one of the main obstacles to the assessment and management of CRF. Therefore, our longitudinal study is designed to build a model by examining longitudinally biopsychosocial correlates of CRF.

The greatest challenge in designing this study was to select the most pertinent and suitable parameters/variables for each of the biopsychosocial dimensions in order to remain feasible for fragile patients. As it is not possible to measure all parameters potentially involved in CRF, we had to exclude some dimensions such as pain or nutrition that would have otherwise been pertinent $[34,70]$. All parameters included are among those most related to CRF, according to the literature.

All patients with localized breast cancer will be included, inherently incorporating various conditions of cancer stages and treatment. Although this may result in a high level of variability and statistical noise, we deemed it necessary to build a real life model that can be later used in clinical practice. Furthermore, with a prevalence up to $50 \%$ for CRF [2], it is reasonable to believe that a proportion of participants will not be suffering from clinically important CRF. This could be perceived as a limitation when studying CRF. However, because clinical relevance is based on cut-off values, some patients could potentially be misclassified and we think that CRF should be understood as a continuum. Moreover, even mild CRF can have an impact on patient quality of life.

The BIOCARE FActory study is expected to highlight the dynamics of CRF and its correlates from diagnosis through post-treatment. SEM analysis will hypothesize relations between latent variables (e.g., physical function), assessed through observed variables (e.g., forcetime asymptote, voluntary activation) and the CRF variable. Results of the present biopsychosocial model will greatly contribute to a better understanding of CRF and its underlying mechanisms, from diagnosis to the aftermaths of cancer and treatments. They could also be applied to improving interventions for CRF management, notably in supportive care, thanks to a better understanding of CRF mechanisms.

\section{Abbreviations \\ CC: Calf Circumference: COP: Centre Of Pressure: CPP: Committee for the Protection of Persons; CRF: Cancer-Related Fatigue; CT: Computed Tomography; ECOG: Eastern Cooperative Oncology Group; EORTC: European Organisation for Research and Treatment of Cancer; $F_{A}$ : Force-time relationship Asymptote; HADS: The Hospital Anxiety and Depression Scale; HR: Heart Rate; IL: Interleukin; LSMI: Lumbar Skeletal Muscle Index; LT1: First Lactate Threshold; MET: Metabolic Equivalent Task; MoCA: Montreal Cognitive Assessment; MVC: Maximal Voluntary Contraction; RPE: Rate of Perceived Exertion; SEM: Structural Equation Modelling; TMT: Trail Making Test; TNF: Tumor Necrosis Factor; VA: Voluntary Activation}

\section{Acknowledgments}

Our thoughts turn particularly to thank Camille Romanet-Faes, who participated in the elaboration of this project and who sadly past away.

\section{Authors' contributions}

$M C, A L, B B, S B, C F, T M, V T O, V P, O Z, L A M, A R$ and $B M$ participated in trial design. $B M$ and $A R$ obtained the funding. $M C, A L, S L, H B$ and $O C$ participated in participants recruitment and to the medical part of the protocol. MC, AR and BM wrote the first draft of the manuscript, which was substantively reviewed by $A L, B B, S B, C F, T M, V T O, V P, O Z, L A M, S L, H B$ and $O C . M C$ reviewed and contributed to the final version of the manuscript. $M C$, $A L, B B, S B, C F, T M, V T O, V P, O Z, L A M, S L, H B, O C, A R$ and $B M$ read and approved the final manuscript. 


\section{Funding}

This study is funded by the 'Ligue contre le cancer' and the 'Région Pays de la Loire'. The funders were not involved in the study design and will not have a role in the data collection, analysis, and interpretation of results or the manuscript.

\section{Availability of data and materials}

Not applicable (the current manuscript contains no data).

\section{Declarations}

\section{Ethics approval and consent to participate}

This study protocol has been approved by the French ethics committee of human research CPP SUD EST VI (IDRCB: 2019-A02525-52) the 13th March 2020. The study is registered on http://www.clinicaltrials.gov (NCT number: NCT04391543). Written informed consent will be obtained from each participant prior their inclusion in the study.

\section{Consent for publication}

Not applicable.

\section{Competing interests}

The authors declare no competing interests.

\section{Author details}

'Le Mans Université, Movement - Interactions - Performance, MIP, 4334, F-72000 Le Mans, EA, France. ${ }^{2}$ Nantes Université, Movement - Interactions Performance, MIP, 4334 Nantes, EA, France. ${ }^{3}$ Elsan-Clinique Victor Hugo, Centre Jean Bernard, Le Mans, France. ${ }^{4}$ Centre Hospitalier Le Mans (CHM), Le Mans, France. ${ }^{5}$ Univ Lyon, University Claude Bernard Lyon 1, Inter-University Laboratory of Human Movement Biology, Team Atherosclerosis Thrombosis \& Physical Activity, EA7424, Lyon, France. 'Le Mans Université, VIPS2, EA4636, Le Mans, France. 'Laboratoire Interuniversitaire de Biologie de la Motricité, Univ. Savoie Mont Blanc, 7424, F-73000 Chambéry, EA, France.

Received: 12 May 2021 Accepted: 4 October 2021

Published online: 23 October 2021

\section{References}

1. Berger AM, Mooney K, Alvarez-Perez A, Breitbart WS, Carpenter KM, Cella D, et al. Cancer-related fatigue, version 2.2015. J Natl Compr Cancer Netw. 2015 Aug;13(8):1012-39. https://doi.org/10.6004/jnccn.2015.0122.

2. Minton O, Stone P. How common is fatigue in disease-free breast cancer survivors? A systematic review of the literature. Breast Cancer Res Treat. 2008 Nov;112(1):5-13. https://doi.org/10.1007/s10549-007-9831-1.

3. Groenvold M, Petersen MA, Idler E, Bjorner JB, Fayers PM, Mouridsen HT. Psychological distress and fatique predicted recurrence and survival in primary breast cancer patients. Breast Cancer Res Treat. 2007 Oct;105(2): 209-19. https://doi.org/10.1007/s10549-006-9447-x.

4. McNeely ML, Courneya KS. Exercise programs for cancer-related fatigue: evidence and clinical guidelines. J Natl Compr Cancer Netw. 2010 Aug;8(8): 945-53. https://doi.org/10.6004/jnccn.2010.0069.

5. Bower JE, Asher A, Garet D, Petersen L, Ganz PA, Irwin MR, et al. Testing a biobehavioral model of fatigue before adjuvant therapy in women with breast cancer. Cancer. 2019 Feb 15;125(4):633-41. https://doi.org/10.1002/ cncr.31827.

6. Weis J. Cancer-related fatigue: prevalence, assessment and treatment strategies. Expert Rev Pharmacoecon Outcomes Res. 2011;11(4):441-6. https://doi.org/10.1586/erp.11.44.

7. Twomey R, Martin T, Temesi J, Culos-Reed SN, Millet GY. Tailored exercise interventions to reduce fatigue in cancer survivors: study protocol of a randomized controlled trial. BMC Cancer. 2018 Jul 24;18(1):757. https://doi. org/10.1186/s12885-018-4668-z.

8. Bower JE. Cancer-related fatigue--mechanisms, risk factors, and treatments. Nat Rev Clin Oncol. 2014 Oct;11(10):597-609. https://doi.org/10.1038/ nrclinonc.2014.127.

9. Andrykowski MA, Schmidt JE, Salsman JM, Beacham AO, Jacobsen PB. Use of a case definition approach to identify cancer-related fatigue in women undergoing adjuvant therapy for breast cancer. J Clin Oncol. 2005 Sep 20; 23(27):6613-22. https://doi.org/10.1200/JCO.2005.07.024.
10. Van Esch L, Roukema JA, Ernst MF, Nieuwenhuijzen GAP, De Vries J. Combined anxiety and depressive symptoms before diagnosis of breast cancer. J Affect Disord. 2012 Feb;136(3):895-901. https://doi.org/10.1016/j.ja d.2011.09.012.

11. Abrahams HJG, Gielissen MFM, Schmits IC, Verhagen CAHHVM, Rovers MM, Knoop H. Risk factors, prevalence, and course of severe fatigue after breast cancer treatment: a meta-analysis involving 12327 breast cancer survivors. Ann Oncol. 2016 Mar 2;27(6):965-74. https://doi.org/10.1093/annonc/ mdw099.

12. Bower JE, Ganz PA, Desmond KA, Rowland JH, Meyerowitz BE, Belin TR. Fatigue in breast cancer survivors: occurrence, correlates, and impact on quality of life. J Clin Oncol. 2000 Feb;18(4):743-53. https://doi.org/10.1200/ JCO.2000.18.4.743.

13. Cella D, Kallich J, McDermott A, Xu X. The longitudinal relationship of hemoglobin, fatigue and quality of life in anemic cancer patients: results from five randomized clinical trials. Ann Oncol. 2004 Jun;15(6):979-86. https://doi.org/10.1093/annonc/mdh235.

14. Collado-Hidalgo A, Bower JE, Ganz PA, Cole SW, Irwin MR. Inflammatory biomarkers for persistent fatigue in breast cancer survivors. Clin Cancer Res. 2006 May 1;12(9):2759-66. https://doi.org/10.1158/1078-0432.CCR-05-2398.

15. Kilgour RD, Vigano A, Trutschnigg B, Hornby L, Lucar E, Bacon SL, et al. Cancer-related fatigue: the impact of skeletal muscle mass and strength in patients with advanced cancer. J Cachexia Sarcopenia Muscle. 2010 Dec 17; 1(2):177-85. https://doi.org/10.1007/s13539-010-0016-0.

16. Yavuzsen T, Davis MP, Ranganathan VK, Walsh D, Siemionow V, Kirkova J, et al. Cancer-related fatigue: central or peripheral? J Pain Symptom Manag. 2009 Oct;38(4):587-96. https://doi.org/10.1016/j.jpainsymman.2008.12.003.

17. Kisiel-Sajewicz K, Davis MP, Siemionow V, Seyidova-Khoshknabi D, Wyant A, Walsh $\mathrm{D}$, et al. Lack of muscle contractile property changes at the time of perceived physical exhaustion suggests central mechanisms contributing to early motor task failure in patients with cancer-related fatigue. J Pain Symptom Manag. 2012 Sep;44(3):351-61. https://doi.org/10.1016/j.jpa insymman.2011.08.007.

18. Kisiel-Sajewicz K, Siemionow V, Seyidova-Khoshknabi D, Davis MP, Wyant A, Ranganathan VK, et al. Myoelectrical manifestation of fatigue less prominent in patients with cancer related fatigue. PLoS One. 2013 Dec 31;8(12):e83636. https://doi.org/10.1371/journal.pone.0083636.

19. Grisold W, Grisold A, Löscher WN. Neuromuscular complications in cancer. J Neurol Sci. 2016 Aug 15;367:184-202. https://doi.org/10.1016/j.jns.2016.06.002.

20. Chartogne M, Rahmani A, Landry S, Bourgeois H, Peyrot N. Neuromuscular, Psychological, and Sleep Predictors of Cancer-Related Fatigue in Cancer Patients. In: Morel B. Psychological, and Sleep Predictors of Cancer-Related Fatigue in Cancer Patients. Clin Breast Cancer: Neuromuscular; 2020 Dec 9.

21. Neil SE, Klika RJ, Garland SJ, McKenzie DC, Campbell KL. Cardiorespiratory and neuromuscular deconditioning in fatigued and non-fatigued breast cancer survivors. Support Care Cancer. 2013 Mar;21(3):873-81. https://doi. org/10.1007/s00520-012-1600-y.

22. Jones LW, Haykowsky MJ, Swartz JJ, Douglas PS, Mackey JR. Early breast cancer therapy and cardiovascular injury. J Am Coll Cardiol. 2007 Oct 9; 50(15):1435-41. https://doi.org/10.1016/j.jacc.2007.06.037.

23. Medysky ME, Temesi J, Culos-Reed SN, Millet GY. Exercise, sleep and cancerrelated fatigue: are they related? Neurophysiol Clin. 2017 Apr 5;47(2):111-22. https://doi.org/10.1016/j.neucli.2017.03.001.

24. Martin T, Twomey R, Medysky ME, Temesi J, Culos-Reed SN, Millet GY. The Relationship between Fatigue and Actigraphy-Derived Sleep and RestActivity Patterns in Cancer Survivors. 2019 Sep 6;

25. Veni T, Boyas S, Beaune B, Bourgeois H, Rahmani A, Landry S, et al. Handgrip fatiguing exercise can provide objective assessment of cancer-related fatigue: a pilot study. Support Care Cancer. 2019 Jan;27(1):229-38. https:// doi.org/10.1007/s00520-018-4320-0.

26. Lockefeer JPM, De Vries J. What is the relationship between trait anxiety and depressive symptoms, fatigue, and low sleep quality following breast cancer surgery? Psychooncology. 2013 May;22(5):1127-33. https://doi.org/1 0.1002/pon.3115.

27. Broeckel JA, Jacobsen PB, Horton J, Balducci L, Lyman GH. Characteristics and correlates of fatigue after adjuvant chemotherapy for breast cancer. J Clin Oncol. 1998 May;16(5):1689-96. https://doi.org/10.1200/JCO.1998.16.5.1689.

28. Spencer KW. Significance of the breast to the individual and society. Plast Surg Nurs. 1996;16(3):131-2. https://doi.org/10.1097/00006527-19962300000003. 
29. Landry S, Chasles G, Pointreau Y, Bourgeois H, Boyas S. Influence of an adapted physical activity program on self-esteem and quality of life of breast Cancer patients after mastectomy. Oncology. 2018 May 30;95(3):18891. https://doi.org/10.1159/000489265.

30. Jenkins V, Thwaites R, Cercignani M, Sacre S, Harrison N, Whiteley-Jones H, et al. A feasibility study exploring the role of pre-operative assessment when examining the mechanism of "chemo-brain" in breast cancer patients. Springerplus. 2016 Mar 31;5(1):390. https://doi.org/10.1186/s40064-016-2030-y.

31. O'Farrell E, MacKenzie J, Collins B. Clearing the air: a review of our current understanding of "chemo fog". Curr Oncol Rep. 2013 Jun;15(3):260-9. https://doi.org/10.1007/s11912-013-0307-7.

32. Prins JB, Bos $E$, Huibers MJH, Servaes $P$, van der Werf SP, van der Meer JWM et al. Social support and the persistence of complaints in chronic fatigue syndrome. Psychother Psychosom. 2004 Jun;73(3):174-82. https://doi.org/1 $0.1159 / 000076455$

33. Fagundes CP, Lindgren ME, Shapiro CL, Kiecolt-Glaser JK. Child maltreatment and breast cancer survivors: social support makes a difference for quality of life, fatigue and cancer stress. Eur J Cancer. 2012 Mar;48(5): 728-36. https://doi.org/10.1016/j.ejca.2011.06.022.

34. Stone P, Richards M, A'Hern R, Hardy J. A study to investigate the prevalence, severity and correlates of fatigue among patients with cancer in comparison with a control group of volunteers without cancer. Ann Oncol. 2000 May;11(5):561-7. https://doi.org/10.1023/A:1008331230608.

35. Humpel N, Iverson DC. Sleep quality, fatigue and physical activity following a cancer diagnosis. Eur J Cancer Care (Engl). 2010 Nov;19(6):761-8. https:// doi.org/10.1111/j.1365-2354.2009.01126.x.

36. De Vries J, Van der Steeg AF, Roukema JA. Trait anxiety determines depressive symptoms and fatigue in women with an abnormality in the breast. Br J Health Psychol. 2009 Feb;14(Pt 1):143-57. https://doi.org/10.134 8/135910708X310200.

37. Courtier N, Gambling T, Enright S, Barrett-Lee P, Abraham J, Mason MD. Psychological and immunological characteristics of fatigued women undergoing radiotherapy for early-stage breast cancer. Support Care Cancer. 2013 Jan;21(1):173-81. https://doi.org/10.1007/s00520-012-1508-6.

38. Brownstein CG, Twomey R, Temesi J, Wrightson JG, Martin T, Medysky ME, et al. Physiological and psychosocial correlates of cancer related fatigue. medRxiv. 2020 Oct 16

39. Jones PR, Pearson J. Anthropometric determination of leg fat and muscle plus bone volumes in young male and female adults. J Physiol Lond. 1969 Oct;204(2):63P-6P

40. Rolland $Y$, Lauwers-Cances $V$, Cournot $M$, Nourhashémi F, Reynish W, Rivière $D$, et al. Sarcopenia, calf circumference, and physical function of elderly women: a cross-sectional study. J Am Geriatr Soc. 2003 Aug;51(8):1120-4. https://doi.org/10.1046/j.1532-5415.2003.51362.x.

41. Arcuri GG, Palladini L, Dumas G, Lemoignan J, Gagnon B. Exploring the measurement properties of the Montreal cognitive assessment in a population of people with cancer. Support Care Cancer. 2015 Sep;23(9): 2779-87. https://doi.org/10.1007/s00520-015-2643-7.

42. Poppelreuter M, Weis J, Külz AK, Tucha O, Lange KW, Bartsch HH. Cognitive dysfunction and subjective complaints of cancer patients. A cross-sectional study in a cancer rehabilitation Centre. Eur J Cancer. 2004 Jan;40(1):43-9. https://doi.org/10.1016/j.ejca.2003.08.001.

43. Campbell KL, Kam JWY, Neil-Sztramko SE, Liu Ambrose T, Handy TC, Lim HJ, et al. Effect of aerobic exercise on cancer-associated cognitive impairment: a proof-of-concept RCT. Psychooncology. 2018;27(1):53-60. https://doi.org/1 0.1002/pon.4370

44. Deschamps $T$, Sauvaget $A$, Pichot $A$, Valrivière $P$, Maroulidès $M$, Bois $A$, et al. Posture-cognitive dual-tasking: a relevant marker of depression-related psychomotor retardation. An illustration of the positive impact of repetitive transcranial magnetic stimulation in patients with major depressive disorder. J Psychiatr Res. 2016 Aug 20;83:86-93. https://doi.org/10.1016/j.jpsychires.2016.08.013.

45. Tucker $\mathrm{R}$. The anticipatory regulation of performance: the physiological basis for pacing strategies and the development of a perception-based model for exercise performance. Br J Sports Med. 2009 Jun;43(6):392-400. https://doi. org/10.1136/bjsm.2008.050799.

46. Burnley M. Estimation of critical torque using intermittent isometric maximal voluntary contractions of the quadriceps in humans. J Appl Physiol. 2009 Mar;106(3):975-83. https://doi.org/10.1152/japplphysiol.91474.2008.

47. Poole DC, Burnley M, Vanhatalo A, Rossiter HB, Jones AM. Critical power: an important fatigue threshold in exercise physiology. Med Sci Sports Exerc 2016;48(11):2320-34. https://doi.org/10.1249/MSS.0000000000000939.
48. Merton PA. Voluntary strength and fatigue. J Physiol Lond. 1954 Mar 29; 123(3):553-64. https://doi.org/10.1113/jphysiol.1954.sp005070.

49. Prado CMM, Lieffers JR, McCargar LJ, Reiman T, Sawyer MB, Martin L, et al. Prevalence and clinical implications of sarcopenic obesity in patients with solid tumours of the respiratory and gastrointestinal tracts: a populationbased study. Lancet Oncol. 2008 Jul;9(7):629-35. https://doi.org/10.1016/S14 70-2045(08)70153-0.

50. Giesinger JM, Kuijpers W, Young T, Tomaszewski KA, Friend E, Zabernigg A, et al. Thresholds for clinical importance for four key domains of the EORTC QLQ-C30: physical functioning, emotional functioning, fatigue and pain. Health Qual Life Outcomes. 2016 Jun 7;14(1):87. https://doi.org/10.1186/s12 955-016-0489-4

51. Kecke S, Ernst J, Einenkel J, Singer S, Hinz A. Psychometric properties of the fatigue questionnaire EORTC QLQ-FA12 in a sample of female Cancer patients. J Pain Symptom Manag. 2017 Aug 12:54(6):922-8. https://doi.org/1 0.1016/j.jpainsymman.2017.08.007.

52. Zigmond AS, Snaith RP. The hospital anxiety and depression scale. Acta Psychiatr Scand. 1983 Jun;67(6):361-70. https://doi.org/10.1111/j.1600-0447.1 983.tb09716.x.

53. Lakens D. Sample Size Justification. 2021 Jan:4.

54. Kline RB. Software review: software programs for structural equation modeling: Amos, EQS, and LISREL. J Psychoeduc Assess. 1998 Dec;16(4):34364. https://doi.org/10.1177/073428299801600407.

55. Grimm LG, Yarnold PR. Reading and understanding MORE multivariate statistics; 2000.

56. Francis DJ. An introduction to structural equation models. J Clin Exp Neuropsychol. 1988 Oct;10(5):623-39. https:/doi.org/10.1080/01688638808402800.

57. Rosseel Y. Lavaan: An R package for structural equation modeling and more. Version 0.5-12 (BETA). J Stat Softw. 2012;

58. Byrne BM. A primer of LISREL: basic applications and programming for confirmatory factor analytic models; 2012.

59. Arbuckle JL, Wothke W. Amos 4.0 user's guide. 1999;

60. Schumacker RE, Lomax RG. A beginner's guide to structural equation modeling. 3rd ed. New York: Routledge; 2010.

61. Tabachnick BG, Fidell LS, Ullman JB. Using multivariate statistics; 2007.

62. Byrne BM. Structural equation modeling with EQS and EQS/windows: basic concepts, applications, and programming; 1994.

63. Steiger $\mathrm{JH}$. Structural model evaluation and modification: an interval estimation approach. Multivariate Behav Res. 1990 Apr 1;25(2):173-80. https://doi.org/10.1207/s15327906mbr2502_4.

64. MacCallum R. Specification searches in covariance structure modeling. Psychol Bull. 1986;100(1):107-20. https://doi.org/10.1037/0033-2909.100.1.107.

65. Bower JE, Bak K, Berger A, Breitbart W, Escalante CP, Ganz PA, et al. Screening, assessment, and management of fatigue in adult survivors of cancer: an American Society of Clinical oncology clinical practice guideline adaptation. J Clin Oncol. 2014 Jun 10;32(17):1840-50. https://doi.org/10.12 00/JCO.2013.53.4495

66. Pearson EJM, Morris ME, McKinstry CE. Cancer-related fatigue: appraising evidence-based guidelines for screening, assessment and management. Support Care Cancer. 2016 Apr 26;24(9):3935-42. https://doi.org/10.1007/ s00520-016-3228-9.

67. Fabi A, Bhargava R, Fatigoni S, Guglielmo M, Horneber M, Roila F, et al. Cancer-related fatigue: ESMO clinical practice guidelines for diagnosis and treatment. Ann Oncol. 2020 Mar 12;31(6):713-23. https://doi.org/10.1016/j.a nnonc.2020.02.016.

68. Seyidova-Khoshknabi D, Davis MP, Walsh D. Review article: a systematic review of cancer-related fatigue measurement questionnaires. Am J Hosp Palliat Care. 2011 Mar;28(2):119-29. https://doi.org/10.1177/1049909110381590.

69. O'Higgins CM, Brady B, O'Connor B, Walsh D, Reilly RB. The pathophysiology of cancer-related fatigue: current controversies. Support Care Cancer. 2018 Oct;26(10):3353-64. https://doi.org/10.1007/s00520-018-4318-7.

70. Norman K, Wirth R, Neubauer M, Eckardt R, Stobäus N. The bioimpedance phase angle predicts low muscle strength, impaired quality of life, and increased mortality in old patients with cancer. J Am Med Dir Assoc. 2015 Feb;16(2):173.e17-22.

\section{Publisher's Note}

Springer Nature remains neutral with regard to jurisdictional claims in published maps and institutional affiliations. 